Research Article

\title{
Some New Explicit Values of Quotients of Ramanujan's Theta Functions and Continued Fractions
}

\author{
Nipen Saikia \\ Department of Mathematics, Rajiv Gandhi University, Rono Hills, Doimukh, Arunachal Pradesh 791112, India \\ Correspondence should be addressed to Nipen Saikia; nipennak@yahoo.com
}

Received 27 December 2013; Accepted 12 May 2014; Published 25 May 2014

Academic Editor: Seppo Hassi

Copyright (C) 2014 Nipen Saikia. This is an open access article distributed under the Creative Commons Attribution License, which permits unrestricted use, distribution, and reproduction in any medium, provided the original work is properly cited.

We evaluate some new explicit values of quotients of Ramanujan's theta functions and use them to find explicit values of Ramanujan's continued fractions.

\section{Introduction}

Ramanujan's general theta function $f(a, b)$ is defined by

$$
f(a, b):=\sum_{k=-\infty}^{\infty} a^{k(k+1) / 2} b^{k(k-1) / 2}, \quad|a b|<1 .
$$

Two important special cases of $f(a, b)$ are the theta functions $\phi(q)$ and $\psi(q)[1$, page 36, Entry 22] defined by, for $|q|<1$,

$$
\begin{aligned}
& \phi(q):=f(q, q)=\sum_{n=-\infty}^{\infty} q^{n^{2}}=\frac{(-q ;-q)_{\infty}}{(q ;-q)_{\infty}}, \\
& \psi(q):=f\left(q, q^{3}\right)=\sum_{n=0}^{\infty} q^{n(n+1) / 2}=\frac{\left(q^{2} ; q^{2}\right)_{\infty}}{\left(q ; q^{2}\right)_{\infty}},
\end{aligned}
$$

where $(a ; q)_{\infty}=\prod_{n=0}^{\infty}\left(1-a q^{n}\right)$.

In his notebooks [2], Ramanujan recorded many explicit values of theta functions $\phi(q)$ and $\psi(q)$. All these values were proved by Berndt [3, page 325] and Berndt and Chan [4]. Yi [5] introduced the parameter $h_{k, n}$ for positive real numbers $k$ and $n$ defined by

$$
h_{k, n}=\frac{\phi(q)}{k^{1 / 4} \phi\left(q^{k}\right)} ; \quad q=e^{-\pi \sqrt{n / k}}
$$

and used the particular case $h_{n, n}$ to find explicit values $\phi(q)$. Baruah and Saikia [6] defined the parameter $g_{k, n}$ for positive real numbers $k$ and $n$ as

$$
g_{k, n}=\frac{\psi(-q)}{k^{1 / 4} q^{(k-1) / 8} \psi\left(-q^{k}\right)} ; \quad q=e^{-\pi \sqrt{n / k}}
$$

and used the particular case $g_{n, n}$ to find explicit values $\psi(q)$. Saikia [7] also established some explicit values $\psi(q)$.

In this paper, we consider the particular cases $h_{3, n}$ and $g_{3, n}$ of the parameters $h_{k, n}$ and $g_{k, n}$, respectively. By using theta function identities, we find some new explicit values of the parameters $h_{3, n}$ and $g_{3, n}$. Particularly, we evaluate $h_{3, n}$ and $g_{3, n}$ for $n=3 / 2,2 / 3,6,1 / 6,5 / 2,2 / 5,10$, and 1/10. Previously, Yi [5] evaluated $h_{3, n}$ for $n=1,3,1 / 3,9,1 / 9,5,1 / 5,25$, and 1/25. Saikia [8] evaluated $h_{3, n}$ for $n=2,1 / 2,4,1 / 4,7,1 / 7$, 49 , and $1 / 49$. Baruah and Saikia [6] evaluated $g_{3, n}$ for $n=1$, $3,1 / 3,9,1 / 9,5,1 / 5,25,1 / 25,7,1 / 7,13,1 / 13,49$, and $1 / 49$. As an application to our new values, we evaluate some old and new explicit values of Ramanujan's cubic continued fraction $G(q)$ and a continued fraction of order twelve $H(q)$ which are, respectively, defined by, for $|q|<1$,

$$
\begin{aligned}
& G(q):=\frac{q^{1 / 3}}{1}+\frac{q+q^{2}}{1}+\frac{q^{2}+q^{4}}{1}+\frac{q^{3}+q^{6}}{1}+\cdots \\
& H(q) \\
& :=\frac{q f\left(-q,-q^{11}\right)}{f\left(-q^{5},-q^{7}\right)} \\
& =\frac{q(1-q)}{1-q^{3}}+\frac{q^{3}\left(1-q^{2}\right)\left(1-q^{4}\right)}{\left(1-q^{3}\right)\left(1+q^{6}\right)}+\frac{q^{3}\left(1-q^{8}\right)\left(1-q^{10}\right)}{\left(1-q^{3}\right)\left(1+q^{12}\right)}+\ldots
\end{aligned}
$$

The continued fraction $G(q)$ was recorded by Ramanujan on page 366 of lost notebook [9]. We refer to [10-14] for explicit evaluations of $G(q)$. The continued fraction $H(q)$ was 
introduced by Naika et al. [15]. We refer to $[8,15]$ for explicit evaluations of $H(q)$.

The presentation of the paper is as follows. In Section 2 we record some preliminary results for ready references in this paper. Section 3 is devoted to explicit evaluations of the parameters $h_{3, n}$ and $g_{3, n}$. In Section 4 we use new explicit values of $h_{3, n}$ and $g_{3, n}$ to evaluate some explicit values of the continued fractions $G(q)$ and $H(q)$.

We end this introduction by noting the following remarks regarding $g_{3, n}$ and $h_{3, n}$ from [6, page 1764, Theorem 4.1] and [5, page 385, Remark 2.3], respectively.

Remark 1. The parameter $g_{3, n}$ has positive real value with $g_{3,1}=1$ and $g_{3, n}$ increases as $n$ increases.

Remark 2. The parameter $h_{3, n}$ has positive real value with $h_{3,1}=1$ and the values of $h_{3, n}$ decrease as $n$ increases.

\section{Preliminary Results}

Lemma 3 (see [16, Theorem 3.2]). If $P:=\sqrt{q} \psi(-q) \psi\left(-q^{9}\right) /$ $\psi^{2}\left(-q^{3}\right)$ and $Q:=q \psi\left(-q^{2}\right) \psi\left(-q^{18}\right) / \psi^{2}\left(-q^{6}\right)$, then

$$
\begin{aligned}
\frac{P^{2}}{Q^{2}}+ & \frac{Q^{2}}{P^{2}}+\left(\frac{P^{2}}{Q^{2}}+\frac{Q^{2}}{P^{2}}\right)^{2} \\
= & \left(3 P Q-\frac{1}{P Q}\right)\left(3 P Q-\frac{1}{P Q}+\frac{3 P}{Q}+\frac{3 Q}{P}+\frac{P^{3}}{Q^{3}}+\frac{Q^{3}}{P^{3}}\right) \\
& +14 .
\end{aligned}
$$

Lemma 4 (see [16, Theorem 3.1]). If $L:=\phi(q) \phi\left(q^{9}\right) / \phi^{2}(q)$ and $M:=\sqrt{q} \psi(-q) \psi\left(-q^{9}\right) / \psi^{2}\left(-q^{3}\right)$, then

$$
L^{2}=\frac{M^{2}+1}{1-3 M^{2}}
$$

Lemma 5 (see [16, Theorem 3.2]). If $P:=\psi\left(-q^{3}\right) \psi\left(-q^{5}\right) /$ $q \psi(-q) \psi\left(-q^{15}\right)$ and $\mathrm{Q}:=\psi\left(-q^{6}\right) \psi\left(-q^{10}\right) / q^{2} \psi\left(-q^{2}\right) \psi\left(-q^{30}\right)$, then

$$
\begin{aligned}
& \left(\frac{P^{2}}{Q^{2}}+\frac{Q^{2}}{P^{2}}\right)+\left(\frac{P}{Q}+\frac{Q}{P}\right)-\left(P Q+\frac{1}{P Q}\right) \\
& +\left(\sqrt{P Q}-\frac{1}{\sqrt{P Q}}\right)\left(\sqrt{\frac{P^{3}}{Q^{3}}}+\sqrt{\frac{Q^{3}}{P^{3}}}+\sqrt{\frac{P}{Q}}+\sqrt{\frac{Q}{P}}\right)=0 .
\end{aligned}
$$

Lemma 6 (see [16, Theorem 3.9]). If $L:=\phi\left(q^{3}\right) \phi\left(q^{5}\right) /$ $\phi(q) \phi\left(q^{15}\right)$ and $M:=\psi\left(-q^{3}\right) \psi\left(-q^{5}\right) / q \psi(-q) \psi\left(-q^{15}\right)$, then

$$
M=\frac{1+L}{1-L} .
$$

Lemma 7 (see [5, page 385, Theorem 2.2]). For all positive real numbers $k$ and $n$, one has

$$
h_{k, 1 / n}=\frac{1}{h_{k, n}} .
$$

Lemma 8 (see [6, page 1764, Theorem 4.1]). For all positive real numbers $k$ and $n$, one has

$$
g_{k, 1 / n}=\frac{1}{g_{k, n}} .
$$

Lemma 9 (see [5, page 393, Theorem 4.9(i)]). For any positive real number $n$, one has

$$
\sqrt{3}\left(h_{3, n} h_{3,9 n}+\frac{1}{h_{3, n} h_{9, n}}\right)=\left(\frac{h_{3,9 n}}{h_{3, n}}\right)^{2}+3
$$

Lemma 10 (see [5, page 394, Theorem 4.12(i)]). For any positive real number $n$, one has

$$
\begin{aligned}
& 3\left\{\left(h_{3, n} h_{3,25 n}\right)^{2}+\frac{1}{\left(h_{3, n} h_{3,25 n}\right)^{2}}\right\} \\
& =\left\{\left(\frac{h_{3,25 n}}{h_{3, n}}\right)^{3}-\left(\frac{h_{3, n}}{h_{3,25 n}}\right)^{3}\right\} \\
& +5\left\{\left(\frac{h_{3,25 n}}{h_{3, n}}\right)^{2}+\left(\frac{h_{3, n}}{h_{3,25 n}}\right)^{2}\right\} \\
& +5\left\{\left(\frac{h_{3,25 n}}{h_{3, n}}\right)-\left(\frac{h_{3, n}}{h_{3,25 n}}\right)\right\} .
\end{aligned}
$$

Lemma 11 (see [6, page 1769, Theorem 5.1(i) and (iii)]). For any positive real number $n$, one has

$$
\text { (i) } \begin{aligned}
& \left(1+\sqrt{3} g_{3, n} g_{3,9 n}\right)^{3}=1+3 g_{3,9 n}^{4}, \\
\text { (ii) } & 3\left\{\left(g_{3, n} g_{3,25 n}\right)^{2}+1 /\left(g_{3, n} g_{3,25 n}\right)^{2}\right\}+5\left\{\left(g_{3,25 n} / g_{3, n}\right)^{2}+\right. \\
& \left.\left(g_{3, n} / g_{3,25 n}\right)^{2}\right\}=\left\{\left(g_{3,25 n} / g_{3, n}\right)^{3}-\left(g_{3, n} / g_{3,25 n}\right)^{3}\right\}+ \\
& 5\left\{\left(g_{3,25 n} / g_{3, n}\right)-\left(g_{3, n} / g_{3,25 n}\right)\right\} .
\end{aligned}
$$

\section{Explicit Evaluations of the Parameters $g_{k, n}$ and $h_{k, n}$}

In this section, we prove some general theorems for the explicit evaluations of the parameters $g_{k, n}$ and $h_{k, n}$ and evaluate some new explicit values therefrom. 
Theorem 12. One has

$$
\begin{aligned}
\left(\frac{g_{3, n} g_{3,36 n}}{g_{3,4 n} g_{3,9 n}}\right)^{2}+\left(\frac{g_{3,4 n} g_{3,9 n}}{g_{3, n} g_{3,36 n}}\right)^{2} \\
+\left(\left(\frac{g_{3, n} g_{3,36 n}}{g_{3,4 n} g_{3,9 n}}\right)^{2}+\left(\frac{g_{3,4 n} g_{3,9 n}}{g_{3, n} g_{3,36 n}}\right)^{2}-14\right. \\
=\left\{3\left(\frac{g_{3, n} g_{3,4 n}}{g_{3,9 n} g_{3,36 n}}\right)-\left(\frac{g_{3,9 n} g_{3,36 n}}{g_{3, n} g_{3,4 n}}\right)\right\} \\
\quad \times\left\{3\left(\frac{g_{3, n} g_{3,4 n}}{g_{3,9 n} g_{3,36 n}}\right)-\left(\frac{g_{3,9 n} g_{3,36 n}}{g_{3, n} g_{3,4 n}}\right)+3\left(\frac{g_{3, n} g_{3,36 n}}{g_{3,4 n} g_{3,9 n}}\right)\right\} \\
+\left\{3\left(\frac{g_{3, n} g_{3,4 n}}{g_{3,9 n} g_{3,36 n}}\right)-\left(\frac{g_{3,9 n} g_{3,36 n}}{g_{3, n} g_{3,4 n}}\right)\right\} \\
\quad \times\left\{3\left(\frac{g_{3,4 n} g_{3,9 n}}{g_{3, n} g_{3,36 n}}\right)+\left(\frac{g_{3, n} g_{3,36 n}}{g_{3,4 n} g_{3,9 n}}\right)^{3}+\left(\frac{g_{3,4 n} g_{3,9 n}}{g_{3, n} g_{3,36 n}}\right)^{3}\right\} .
\end{aligned}
$$

Proof. We set $q:=e^{-\pi \sqrt{n / 3}}$ in Lemma 3 and use the definition of $g_{3, n}$.

\section{Corollary 13. One has}

$$
\begin{gathered}
g_{3,3 / 2}=\frac{(\sqrt{3 \sqrt{2+2 \sqrt{2}}-\sqrt{6+6 \sqrt{2}}})}{\sqrt{2}}, \\
g_{3,6}=\frac{\sqrt{6(1+\sqrt{2})}}{\sqrt{3 \sqrt{2+2 \sqrt{2}}-\sqrt{6+6 \sqrt{2}}}}, \\
g_{3,2 / 3}=\frac{\sqrt{2}}{(\sqrt{3 \sqrt{2+2 \sqrt{2}}-\sqrt{6+6 \sqrt{2}}})}, \\
g_{3,6}=\frac{\sqrt{3 \sqrt{2+2 \sqrt{2}}-\sqrt{6+6 \sqrt{2}}}}{\sqrt{6(1+\sqrt{2})}} .
\end{gathered}
$$

Proof. Setting $n=1 / 6$ in Theorem 12 and simplifying using Lemma 8 , we obtain

$$
\begin{aligned}
& \left(\frac{3}{\left(g_{3,3 / 2} g_{3,6}\right)^{2}}-\left(g_{3,3 / 2} g_{3,6}\right)^{2}\right) \\
& \quad \times\left(\frac{3}{\left(g_{3,3 / 2} g_{3,6}\right)^{2}}-\left(g_{3,3 / 2} g_{3,6}\right)^{2}+8\right)+8=0 .
\end{aligned}
$$

Solving (16) for $g_{3,3 / 2} g_{3,6}$ and noting the fact in Remark 1 , we find that

$$
g_{3,3 / 2} g_{3,6}=\sqrt{3(1+\sqrt{2})}
$$

Again, setting $n=1 / 6$ in Lemma $11(\mathrm{i})$ and simplifying using Lemma 8, we deduce that

$$
\left(1+\sqrt{3}\left(\frac{g_{3,3 / 2}}{g_{3,6}}\right)\right)^{3}=1+3 g_{3,3 / 2}^{4} .
$$

Eliminating $g_{3,6}$ from (18) using (17) and simplifying, we obtain

$$
g_{3,3 / 2}^{4}+3\left(\sqrt{1+\sqrt{2}}-(1+\sqrt{2})^{3 / 2}\right) g_{3,3 / 2}^{2}+(3+3 \sqrt{2})=0
$$

Solving (19) and noting the fact in Remark 1, we obtain

$$
g_{3,3 / 2}=\frac{(\sqrt{3 \sqrt{2+2 \sqrt{2}}-\sqrt{6+6 \sqrt{2}}})}{\sqrt{2}} .
$$

Employing (20) in (17) and simplifying, we obtain

$$
g_{3,6}=\frac{\sqrt{6(1+\sqrt{2})}}{\sqrt{3 \sqrt{2+2 \sqrt{2}}-\sqrt{6+6 \sqrt{2}}}} .
$$

Now the values of $g_{3,2 / 3}$ and $g_{3,1 / 6}$ follow from the values of $g_{3,3 / 2}$ and $g_{3,6}$, respectively, and Lemma 8 .

Theorem 14. One has

$$
\left(\frac{h_{3, n}}{h_{3,9 n}}\right)^{2}\left\{1-3\left(\frac{g_{3, n}}{g_{3,9 n}}\right)^{2}\right\}-\left(\frac{g_{3, n}}{g_{3,9 n}}\right)^{2}-1=0 .
$$

Proof. We set $q:=e^{-\pi \sqrt{n / 3}}$ in Lemma 4 and use the definitions of $h_{3, n}$ and $g_{3, n}$.

Corollary 15. One has

$$
\begin{gathered}
h_{3,3 / 2}=2^{-5 / 8}(3 \sqrt{2}-4)^{3 / 4}(6+6 \sqrt{2}+\sqrt{6})^{1 / 2}, \\
h_{3,6}=2^{1 / 8} 3^{1 / 2}(3 \sqrt{2}+4)^{1 / 4}(6+6 \sqrt{2}+\sqrt{6})^{-1 / 2}, \\
h_{3,2 / 3}=2^{5 / 8}(3 \sqrt{2}-4)^{-3 / 4}(6+6 \sqrt{2}+\sqrt{6})^{-1 / 2}, \\
h_{3,1 / 6}=2^{-1 / 8} 3^{-1 / 2}(3 \sqrt{2}+4)^{-1 / 4}(6+6 \sqrt{2}+\sqrt{6})^{1 / 2} .
\end{gathered}
$$

Proof. Setting $n=1 / 6$ in Theorem 14 and simplifying using Lemma 7, we obtain

$$
\frac{1}{\left(h_{3,6} h_{3,3 / 2}\right)^{2}}\left(1-\frac{3}{\left(g_{3,6} g_{3,3 / 2}\right)^{2}}\right)-\frac{1}{\left(g_{3,6} g_{3,3 / 2}\right)^{2}}-1=0 .
$$

Employing (17) in (24), solving the resulting equation, and noting the fact in Remark 2, we deduce that

$$
h_{3,3 / 2} h_{3,6}=2^{1 / 4} \sqrt{\frac{3}{4+3 \sqrt{2}}} .
$$


Again setting $n=1 / 6$ in Lemma 9 and simplifying using Lemma 7, we obtain

$$
\sqrt{3}\left(\frac{h_{3,3 / 2}}{h_{3,6}}+\frac{h_{3,6}}{h_{3,3 / 2}}\right)=\left(h_{3,3 / 2} h_{3,6}\right)^{2}+3 .
$$

Employing (25) in (26), solving the resulting equation, and noting the fact in Remark 2, we deduce that

$$
\frac{h_{3,3 / 2}}{h_{3,6}}=\frac{6+6 \sqrt{2}+\sqrt{6}}{\sqrt{3}(4+3 \sqrt{2})} .
$$

Multiplying (25) and (27), we evaluate the value of $h_{3,3 / 2}$, and dividing (25) by (27) and simplifying, we evaluate the value of $h_{3,6}$. Now the values of $h_{3,2 / 3}$ and $h_{3,1 / 6}$ easily follow from the values of $h_{3,3 / 2}$ and $h_{3,6}$, respectively, and Lemma 7 .

Theorem 16. One has

$$
\begin{gathered}
\left(\frac{g_{3,4 n} g_{3,25 n}}{g_{3, n} g_{3,100 n}}\right)^{2}+\left(\frac{g_{3, n} g_{3,100 n}}{g_{3,4 n} g_{3,25 n}}\right)^{2}+\left(\frac{g_{3,4 n} g_{3,25 n}}{g_{3, n} g_{3,100 n}}\right) \\
+\left(\frac{g_{3, n} g_{3,100 n}}{g_{3,4 n} g_{3,25 n}}\right)-\left(\frac{g_{3,25 n} g_{3,100 n}}{g_{3, n} g_{3,4 n}}\right)-\left(\frac{g_{3, n} g_{3,4 n}}{g_{3,25 n} g_{3,100 n}}\right) \\
+\left\{\left(\frac{g_{3,25 n} g_{3,100 n}}{g_{3, n} g_{3,4 n}}\right)^{1 / 2}-\left(\frac{g_{3, n} g_{3,4 n}}{g_{3,25 n} g_{3,100 n}}\right)^{1 / 2}\right\} \\
\times\left\{\left(\frac{g_{3,4 n} g_{3,25 n}}{g_{3, n} g_{3,100 n}}\right)^{3 / 2}+\left(\frac{g_{3, n} g_{3,100 n}}{g_{3,4 n} g_{3,25 n}}\right)^{3 / 2}\right. \\
\left.+\left(\frac{g_{3,4 n} g_{3,25 n}}{g_{3, n} g_{3,100 n}}\right)^{1 / 2}+\left(\frac{g_{3, n} g_{3,100 n}}{g_{3,4 n} g_{3,25 n}}\right)^{1 / 2}\right\}=0 .
\end{gathered}
$$

Proof. We set $q:=e^{-\pi \sqrt{n / 3}}$ in Lemma 5 and use the definition of $g_{3, n}$.

\section{Corollary 17. One has}

$$
\begin{aligned}
& g_{3,5 / 2}=(1+\sqrt{3}+\sqrt{2+\sqrt{3}})^{1 / 2}(a+\sqrt{5 b})^{1 / 4} c^{-1 / 4}, \\
& g_{3,10}=(1+\sqrt{3}+\sqrt{2+\sqrt{3}})^{1 / 2}(a+\sqrt{5 b})^{-1 / 4} c^{1 / 4}, \\
& g_{3,2 / 5}=(1+\sqrt{3}+\sqrt{2+\sqrt{3}})^{-1 / 2}(a+\sqrt{5 b})^{-1 / 4} c^{1 / 4}, \\
& g_{3,1 / 10}=(1+\sqrt{3}+\sqrt{2+\sqrt{3}})^{-1 / 2}(a+\sqrt{5 b})^{1 / 4} c^{-1 / 4},
\end{aligned}
$$

where

$$
\begin{aligned}
& a=63+35 \sqrt{3}+30 \sqrt{2+\sqrt{3}}+20 \sqrt{6+3 \sqrt{3}} \\
& b=2574+1485 \sqrt{3}+1330 \sqrt{2+\sqrt{3}}+770 \sqrt{6+3 \sqrt{3}} \\
& c=25+15 \sqrt{3}+14 \sqrt{2+\sqrt{3}}+7 \sqrt{6+3 \sqrt{3}}
\end{aligned}
$$

Proof. Setting $n=1 / 10$ in Theorem 16 and simplifying using Lemma 8, we obtain

$$
\begin{aligned}
& \left(g_{3,5 / 2} g_{3,10}\right)^{2}+\frac{1}{\left(g_{3,5 / 2} g_{3,10}\right)^{2}} \\
& \quad-4\left(g_{3,5 / 2} g_{3,10}-\frac{1}{g_{3,5 / 2} g_{3,10}}\right)-4=0 .
\end{aligned}
$$

Solving (31) and noting the fact in Remark 2, we find that

$$
g_{3,5 / 2} g_{3,10}=1+\sqrt{3}+\sqrt{2+\sqrt{3}}
$$

Again, setting $n=1 / 10$ in Lemma 11 (ii) and simplifying using Lemma 8, we obtain

$$
\begin{aligned}
& 3\left\{\left(\frac{g_{3,5 / 2}}{g_{3,10}}\right)^{2}+\left(\frac{g_{3,5 / 2}}{g_{3,10}}\right)\right\} \\
& +5\left(\left(g_{3,5 / 2} g_{3,10}\right)^{2}+\frac{1}{\left(g_{3,5 / 2} g_{3,10}\right)^{2}}\right) \\
& -\left(\left(g_{3,5 / 2} g_{3,10}\right)^{3}-\frac{1}{\left(g_{3,5 / 2} g_{3,10}\right)^{3}}\right) \\
& -5\left(g_{3,5 / 2} g_{3,10}-\frac{1}{g_{3,5 / 2} g_{3,10}}\right)=0 .
\end{aligned}
$$

Employing (32) in (33), solving the resulting equation, and noting the fact in Remark 1, we obtain

$$
\frac{g_{3,10}}{g_{3,5 / 2}}=\left(\frac{a+\sqrt{5 b}}{c}\right)^{1 / 2},
$$

where $a=63+35 \sqrt{3}+30 \sqrt{2+\sqrt{3}}+20 \sqrt{6+3 \sqrt{3}}, b=2574+$ $1485 \sqrt{3}+1330 \sqrt{2+\sqrt{3}}+770 \sqrt{6+3 \sqrt{3}}$, and $c=25+15 \sqrt{3}+$ $14 \sqrt{2+\sqrt{3}}+7 \sqrt{6+3 \sqrt{3}}$.

Combining (32) and (34), we calculate the values of $g_{3,5 / 2}$ and $g_{3,10}$. Then, the values of $g_{3,2 / 5}$ and $g_{3,1 / 10}$ follow from the values of $g_{3,5 / 2}$ and $g_{3,10}$, respectively, and Lemma 8 .

Theorem 18. One has

$$
\left(\frac{g_{3,25 n}}{g_{3, n}}\right)\left\{1-\left(\frac{h_{3,25 n}}{h_{3, n}}\right)\right\}-1-\left(\frac{h_{3,25 n}}{h_{3, n}}\right)=0
$$

Proof. We set $q:=e^{-\pi \sqrt{n / 3}}$ in Lemma 6 and use the definitions of $h_{3, n}$ and $g_{3, n}$. 
Corollary 19. One has

$$
\begin{aligned}
h_{3,5 / 2}= & (\sqrt{3}+\sqrt{2+\sqrt{3}})^{1 / 2}(2+\sqrt{3}+\sqrt{2+\sqrt{3}})^{-1 / 2} \\
& \times(f-4 \sqrt{5 b})^{1 / 4} d^{-1 / 4} \\
h_{3,10}= & (\sqrt{3}+\sqrt{2+\sqrt{3}})^{1 / 2}(2+\sqrt{3}+\sqrt{2+\sqrt{3}})^{-1 / 2} \\
& \times(f-4 \sqrt{5 b})^{-1 / 4} d^{1 / 4}, \\
h_{3,2 / 5}= & (\sqrt{3}+\sqrt{2+\sqrt{3}})^{-1 / 2}(2+\sqrt{3}+\sqrt{2+\sqrt{3}})^{1 / 2} \\
& \times(f-4 \sqrt{5 b})^{-1 / 4} d^{1 / 4}, \\
h_{3,1 / 10}= & (\sqrt{3}+\sqrt{2+\sqrt{3}})^{-1 / 2}(2+\sqrt{3}+\sqrt{2+\sqrt{3}})^{1 / 2} \\
& \times(f-4 \sqrt{5 b})^{1 / 4} d^{-1 / 4},
\end{aligned}
$$

where $b$ is given in Corollary 17, $f=1143+655 \sqrt{3}+$ $582 \sqrt{2+\sqrt{3}}+346 \sqrt{6+3 \sqrt{3}}$, and $d=1117+645 \sqrt{3}+$ $578 \sqrt{2+\sqrt{3}}+334 \sqrt{6+3 \sqrt{3}}$.

Proof. Setting $n=1 / 10$ in Theorem 18 and simplifying using Lemma 7, we get

$$
g_{3,5 / 2} g_{3,10}\left(1-h_{3,5 / 2} h_{3,10}\right)-1-h_{3,5 / 2} h_{3,10}=0 .
$$

Employing (32) and solving the resulting equation, we obtain

$$
h_{3,5 / 2} h_{3,10}=\frac{\sqrt{3}+\sqrt{2+\sqrt{3}}}{2+\sqrt{3}+\sqrt{2+\sqrt{3}}} .
$$

Again setting $n=1 / 10$ in Lemma 10 and simplifying using Lemma 7 , we find that

$$
\begin{gathered}
3\left\{\left(\frac{h_{3,5 / 2}}{h_{3,10}}\right)^{2}+\left(\frac{h_{3,10}}{h_{3,5 / 2}}\right)^{2}\right\} \\
-\left(\left(h_{3,5 / 2} h_{3,10}\right)^{3}-\frac{1}{\left(h_{3,5 / 2} h_{3,10}\right)^{3}}\right) \\
-5\left(\left(h_{3,5 / 2} h_{3,10}\right)^{2}+\frac{1}{\left(h_{3,5 / 2} h_{3,10}\right)^{2}}\right) \\
-5\left(h_{3,5 / 2} h_{3,10}-\frac{1}{h_{3,5 / 2} h_{3,10}}\right)=0 .
\end{gathered}
$$

Employing (38) in (39), solving the resulting equation, and noting the fact in Remark 2, we obtain

$$
\frac{h_{3,5 / 2}}{h_{3,10}}=\sqrt{\frac{f-4 \sqrt{5 b}}{d}},
$$

where $b$ is given in Corollary 17, $f=1143+655 \sqrt{3}+$ $582 \sqrt{2+\sqrt{3}}+346 \sqrt{6+3 \sqrt{3}}$, and $d=1117+645 \sqrt{3}+$ $578 \sqrt{2+\sqrt{3}}+334 \sqrt{6+3 \sqrt{3}}$.

Combining (38) and (40), we evaluate the values of $h_{3,5 / 2}$ and $h_{3,10}$. Then the values of $h_{3,2 / 5}$ and $h_{3,1 / 10}$ follow from $h_{3,5 / 2}$ and $h_{3,10}$, respectively, and Lemma 7 .

\section{Explicit Evaluations of Continued Fractions}

This section is devoted to finding some explicit values of the continued fractions $G(q)$ and $H(q)$ by using new values of $g_{3, n}$ and $h_{3, n}$ evaluated in Section 3 .

Lemma 20 (see [6, page 1788, Theorem 9.1(i)]). One has

$$
G^{3}\left(e^{-\pi \sqrt{n / 3}}\right)=\frac{-1}{1+3 g_{3, n}^{4}} .
$$

Theorem 21. One has

$$
\begin{gathered}
G^{3}\left(-e^{-\pi / \sqrt{2}}\right)=\frac{2}{9 \sqrt{36+42 \sqrt{2}}-(38+36 \sqrt{2})}, \\
G^{3}\left(-e^{-\pi \sqrt{2}}\right) \\
=\frac{(3 \sqrt{2+2 \sqrt{2}}-\sqrt{6+6 \sqrt{2}})^{2}}{6(\sqrt{(2+2 \sqrt{2})(6+6 \sqrt{2})}-(58+40 \sqrt{2}))}, \\
G^{3}\left(-e^{-\pi \sqrt{2} / 3}\right) \\
G^{3}\left(-e^{-\pi / 3 \sqrt{2}}\right)=\frac{(3 \sqrt{2+2 \sqrt{2}}-\sqrt{6+6 \sqrt{2}})^{2}}{6(\sqrt{(2+2 \sqrt{2})(6+6 \sqrt{2})}-(6+4 \sqrt{2}))}, \\
G^{3}\left(-e^{-\pi \sqrt{5 / 6}}\right)=\frac{-c(1+\sqrt{2})}{c+3(a+\sqrt{5 b})(1+\sqrt{3}+\sqrt{2+\sqrt{3}})^{2}}, \\
G^{3}\left(-e^{-\pi / \sqrt{30}}\right)=\frac{-(a+\sqrt{5 b})}{c(1+\sqrt{3}+\sqrt{2+\sqrt{3}})^{2}+3(a+\sqrt{5 b})}, \\
G^{3}\left(-e^{-\pi \sqrt{10 / 3}}\right)=\frac{-(a+\sqrt{5 b})(1+\sqrt{3}+\sqrt{2+\sqrt{3}})^{2}}{(a+\sqrt{5 b})+3 c(1+\sqrt{3}+\sqrt{2+\sqrt{3}})^{2}},
\end{gathered}
$$

where $a, b$, and $c$ are given in Corollary 17 . 
Proof. We set $n=3 / 2,6,2 / 3,1 / 6,5 / 2,10,2 / 5$, and $1 / 10$ in Lemma 20 and use the corresponding values of $g_{3, n}$ from Corollaries 13 and 17 to complete the proof.

The explicit values of $G^{3}\left(-e^{-\pi / 3 \sqrt{2}}\right), G^{3}\left(-e^{-\pi \sqrt{5 / 6}}\right)$, $G^{3}\left(-e^{-\pi \sqrt{10 / 3}}\right), G^{3}\left(-e^{-\pi \sqrt{2 / 15}}\right)$, and $G^{3}\left(-e^{-\pi / \sqrt{30}}\right)$ are new.

Lemma 22 (see [8, page 144, Theorem 5.1]). One has

$$
H\left(e^{-\pi \sqrt{n / 3}}\right)=\frac{3^{1 / 4} h_{3, n}-1}{3^{1 / 4} h_{3, n}+1} .
$$

By setting $n=3 / 2,6,2 / 3,1 / 6,5 / 2,10,2 / 5$, and $1 / 10$ in Lemma 22 and employing the corresponding values of $h_{3, n}$ from Corollaries 15 and 19, we calculate the following new explicit values of the continued fraction $H(q)$.

Theorem 23. One has

$$
\begin{aligned}
& H\left(e^{-\pi / \sqrt{2}}\right)=\frac{-2+2^{3 / 8} 3^{1 / 4}(3 \sqrt{2}-4)^{3 / 4} \sqrt{1+6 \sqrt{2}+\sqrt{6}}}{2+2^{3 / 8} 3^{1 / 4}(3 \sqrt{2}-4)^{3 / 4} \sqrt{1+6 \sqrt{2}+\sqrt{6}}}, \\
& H\left(e^{-\pi \sqrt{2}}\right)=\frac{2^{1 / 8} 3^{3 / 4}(4+3 \sqrt{2})^{1 / 4}-\sqrt{1+6 \sqrt{2}+\sqrt{6}}}{2^{1 / 8} 3^{3 / 4}(4+3 \sqrt{2})^{1 / 4}+\sqrt{1+6 \sqrt{2}+\sqrt{6}}} \\
& H\left(e^{-\pi \sqrt{2} / 3}\right)=\frac{2^{5 / 8} 3^{1 / 4}-(3 \sqrt{2}-4)^{3 / 4} \sqrt{1+6 \sqrt{2}+\sqrt{6}}}{2^{5 / 8} 3^{1 / 4}+(3 \sqrt{2}-4)^{3 / 4} \sqrt{1+6 \sqrt{2}+\sqrt{6}}} \\
& H\left(e^{-\pi / \sqrt{18}}\right)=\frac{2^{7 / 8} \sqrt{1+6 \sqrt{2}+\sqrt{6}}-2(12+9 \sqrt{2})^{1 / 4}}{2^{7 / 8} \sqrt{1+6 \sqrt{2}+\sqrt{6}}+2(12+9 \sqrt{2})^{1 / 4}}, \\
& H\left(e^{-\pi \sqrt{5 / 6}}\right) \\
& =\left(3^{1 / 4}(3+\sqrt{2+\sqrt{3}})^{1 / 2}(f-4 \sqrt{5 b})^{1 / 4}\right. \\
& \left.-(2+\sqrt{3}+\sqrt{2+\sqrt{3}})^{1 / 2} d^{1 / 4}\right) \\
& \times\left(3^{1 / 4}(3+\sqrt{2+\sqrt{3}})^{1 / 2}(f-4 \sqrt{5 b})^{1 / 4}\right. \\
& \left.+(2+\sqrt{3}+\sqrt{2+\sqrt{3}})^{1 / 2} d^{1 / 4}\right)^{-1}
\end{aligned}
$$

$$
\begin{aligned}
& H\left(e^{-\pi \sqrt{10 / 3}}\right) \\
& =\left(3^{1 / 4}(3+\sqrt{2+\sqrt{3}})^{1 / 2} d^{1 / 4}\right. \\
& \left.-(2+\sqrt{3}+\sqrt{2+\sqrt{3}})^{1 / 2}(f-4 \sqrt{5 b})^{1 / 4}\right) \\
& \times\left(3^{1 / 4}(3+\sqrt{2+\sqrt{3}})^{1 / 2} d^{1 / 4}\right. \\
& \left.+(2+\sqrt{3}+\sqrt{2+\sqrt{3}})^{1 / 2}(f-4 \sqrt{5 b})^{1 / 4}\right)^{-1} \\
& H\left(e^{-\pi \sqrt{2 / 15}}\right) \\
& =\left(3^{1 / 4}(2+\sqrt{3}+\sqrt{2+\sqrt{3}})^{1 / 2} d^{1 / 4}\right. \\
& \left.-(3+\sqrt{2+\sqrt{3}})^{1 / 2}(f-4 \sqrt{5 b})^{1 / 4}\right) \\
& \times\left(3^{1 / 4}(2+\sqrt{3}+\sqrt{2+\sqrt{3}})^{1 / 2} d^{1 / 4}\right. \\
& \left.+(3+\sqrt{2+\sqrt{3}})^{1 / 2}(f-4 \sqrt{5 b})^{1 / 4}\right)^{-1}, \\
& H\left(e^{-\pi / \sqrt{30}}\right) \\
& =\left(3^{1 / 4}(2+\sqrt{3}+\sqrt{2+\sqrt{3}})^{1 / 2}(f-4 \sqrt{5 b})^{1 / 4}\right. \\
& \left.-(3+\sqrt{2+\sqrt{3}})^{1 / 2} d^{1 / 4}\right) \\
& \times\left(3^{1 / 4}(2+\sqrt{3}+\sqrt{2+\sqrt{3}})^{1 / 2}(f-4 \sqrt{5 b})^{1 / 4}\right. \\
& \left.+(3+\sqrt{2+\sqrt{3}})^{1 / 2} d^{1 / 4}\right)^{-1}
\end{aligned}
$$

where $b$ is given in Corollary 17 and $d$ and $f$ are given in Corollary 19.

\section{Conflict of Interests}

The author declares that there is no conflict of interests regarding the publication of this paper. 


\section{Acknowledgment}

The author is thankful to the University Grants Commission, New Delhi, India, for partially supporting the research work under Grant F. no. 41-1394/2012(SR).

\section{References}

[1] B. C. Berndt, Ramanujan's Notebooks, Springer, New York, NY, USA, 1991.

[2] S. Ramanujan, Notebooks, Tata Institute of Fundamental Research, Bombay, India, 1957.

[3] B. C. Berndt, Ramanujan's Notebooks, Springer, New York, NY, USA, 1998.

[4] B. C. Berndt and H. H. Chan, "Ramanujan's explicit values for the classical theta-functions," Mathematika, vol. 42, no. 2, pp. 278-294, 1995.

[5] J. Yi, "Theta-function identities and the explicity formulas for theta-function and their applications," Journal of Mathematical Analysis and Applications, vol. 292, no. 2, pp. 381-400, 2004.

[6] N. D. Baruah and N. Saikia, "Two parameters for Ramanujan's theta-functions and their explicit values," Rocky Mountain Journal of Mathematics, vol. 37, no. 6, pp. 1747-1790, 2007.

[7] N. Saikia, "A new parameter for Ramanujan's theta-functions and explicit values," Arab Journal of Mathematical Sciences, vol. 18, no. 2, pp. 105-119, 2012.

[8] N. Saikia, "Modular identities and explicit values of a continued fraction of order twelve," JP Journal of Algebra, Number Theory and Applications, vol. 22, no. 2, pp. 127-154, 2011.

[9] S. Ramanujan, The Lost Notebook and Other Unpublished Papers, Narosa, New Delhi, India, 1988.

[10] C. Adiga, M. S. M. Naika, and K. R. Vasuki, "Some new explicit evaluations for Ramanujan's cubic continued fraction," New Zealand Journal of Mathematics, vol. 31, no. 2, pp. 109-114, 2002.

[11] C. Adiga, T. Kim, M. S. M. Naika, and H. S. Madhusudhan, "On Ramanujan's cubic continued fraction and explicit evaluations of theta-functions," Indian Journal of Pure and Applied Mathematics, vol. 35, no. 9, pp. 1047-1062, 2004.

[12] N. D. Baruah and N. Saikia, "Some general theorems on the explicit evaluations of Ramanujan's cubic continued fraction," Journal of Computational and Applied Mathematics, vol. 160, no. 1-2, pp. 37-51, 2003.

[13] B. C. Berndt, H. H. Chan, and L.-C. Zhang, "Ramanujan's class invariants and cubic continued fraction," Acta Arithmetica, vol. 73, pp. 67-85, 1995.

[14] H. H. Chan, “On Ramanujan's cubic continued fraction," Acta Arithmetica, vol. 73, pp. 343-355, 1995.

[15] M. S. M. Naika, B. N. Dharmendra, and K. Shivashankara, "A continued fraction of order twelve," Central European Journal of Mathematics, vol. 6, no. 3, pp. 393-404, 2008.

[16] M. S. M. Naika, S. Chandankumar, and M. Harish, "On some new mixed modular equations involving Ramanujan's thetafunctions," Matematicki Vesnik, vol. 66, no. 3, pp. 283-293, 2014. 


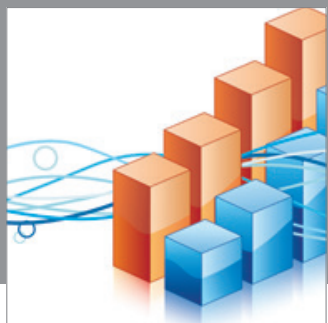

Advances in

Operations Research

mansans

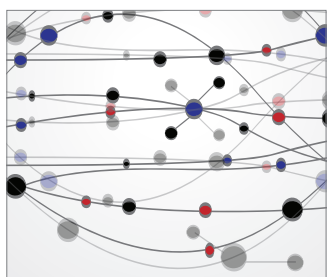

The Scientific World Journal
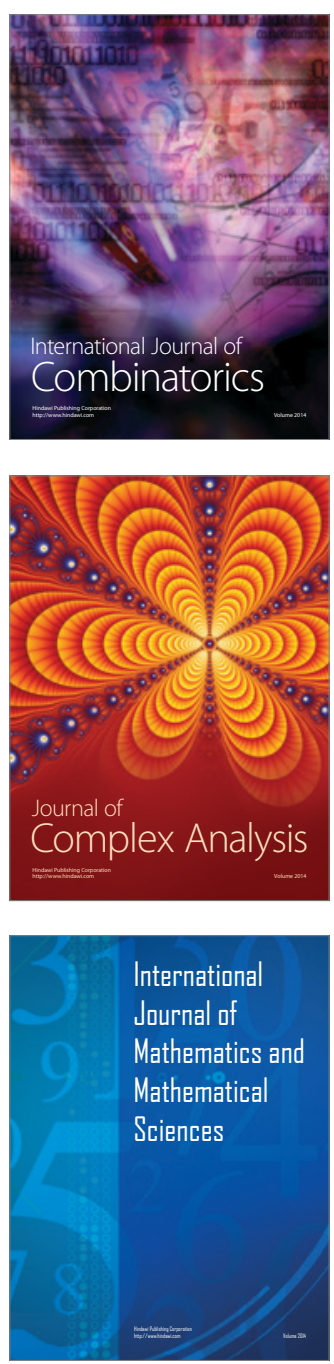
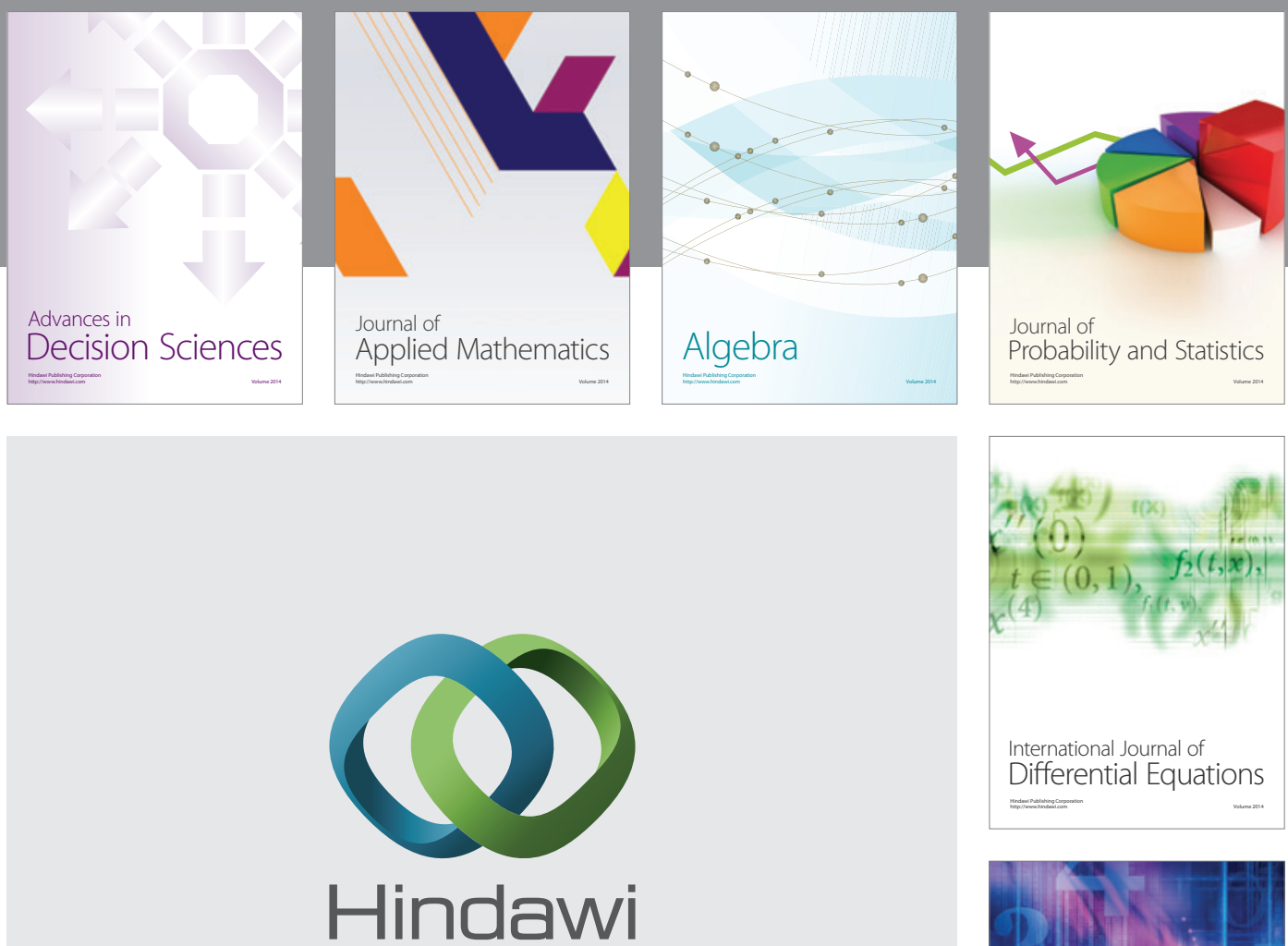

Submit your manuscripts at http://www.hindawi.com
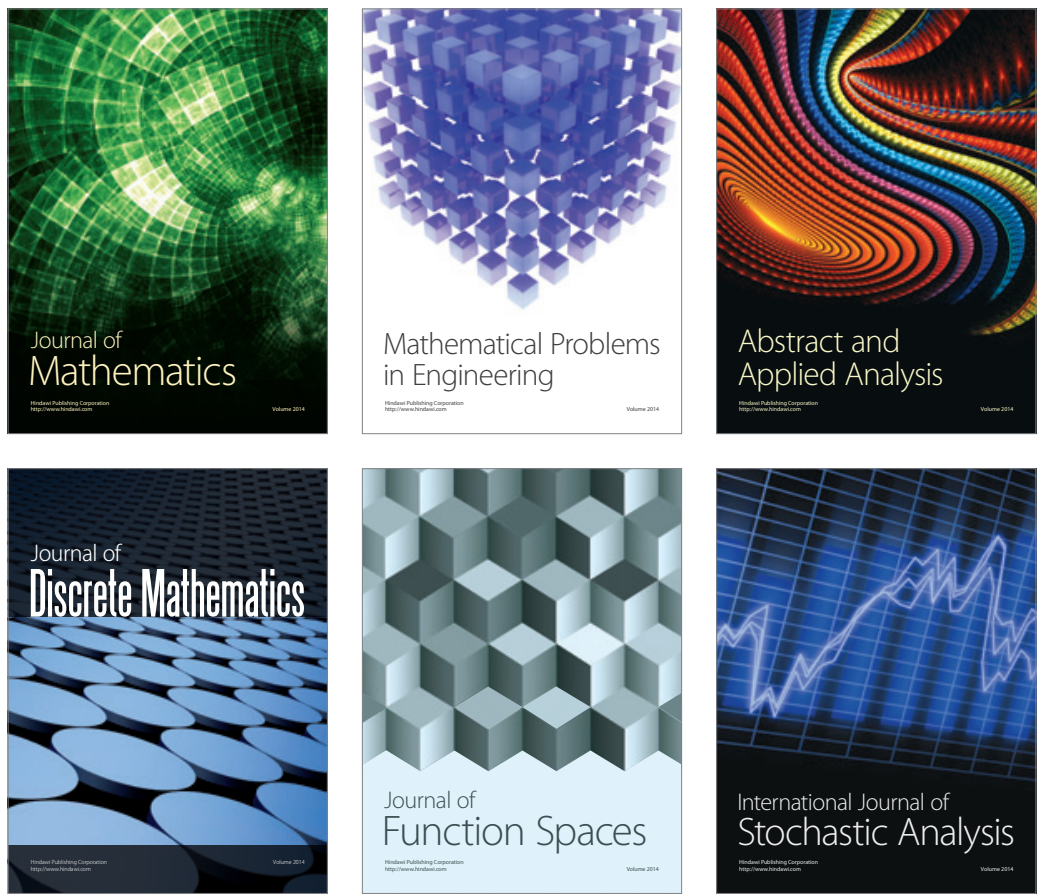

Journal of

Function Spaces

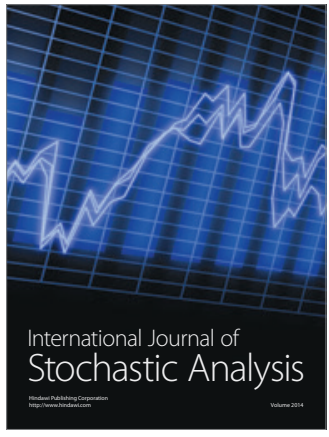

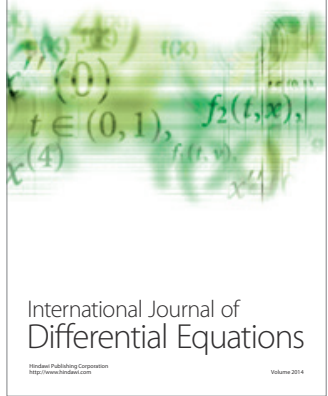
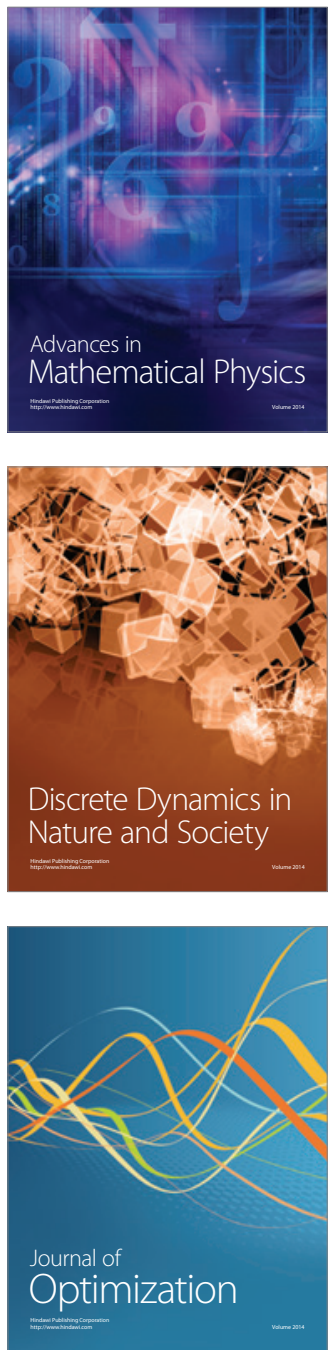